\title{
What is 'Lean'? An overview and its emerging role in health care
}

\author{
Douglas R McKay MD MBA FRCSC ${ }^{1}$, Daniel A Peters MD MBA FRCSC ${ }^{2}$
}

I t seems that, as each day passes, surgeons in Canada are expected to better understand and control the cost implications of their craft and its impact on the broader system. As budgets continue to contract relative to inflation and previous epochs of prosperity, austerity and cost containment will continue to dominate health care delivery. Cost control is not a novel concept for businesses that are obliged to answer to principals' and shareholders' demands for profit. Health care in Canada has remained relatively impervious to this metric, where the notion of patient care has always trumped penny pinching. In fact, perseverating over cost in the past and choosing lower cost alternatives was, in a way, akin to offering inferior care. The pendulum has swung; cost containment is regarded as a means to system salvation and self-preservation.

As cost control migrated into the Canadian health care system, cost containment principles and systems have slowly followed. Health care providers must become facile with both the language and the philosophies at play.

'Lean' is one of those terms that seems to get a great deal of airtime at administrative meetings. Its definition and the details of its execution are nebulous to many and are rarely, if ever, explained. At times, the more critical among us may wonder whether those who use the term entirely understand its underlying principles. It is commonly assumed that lean is all about cost, but it is just as much about quality. It is boardroom and monthly meeting banter, thrown around to a sea of nodding heads. All agree that a lean approach to system and process streamlining is imperative, but what is lean and how can it help our beleaguered health care system?

\section{THE ROOTS OF LEAN: \\ AUTOMOBILE ASSEMBLY LINES AT TOYOTA}

To understand lean as applied to health care, one must first appreciate its lineage. The term 'lean' was coined in the late 1980s, but the manufacturing management philosophy that it glorifies finds its roots in the Japanese auto manufacturing industry. The culture began to shift here toward lean processing as early as the 1930s and its integration ballooned in the years following WWII. Lean was created to improve the efficiency of assembly lines. More specifically, lean was created to minimize waste while answering to customer value. Manufacturing costs fall and productivity and profit increase when unnecessary waste is shed.

\section{A focus on value}

Lean tweaks the manufacturing supply chain to answer to the customer's perception of value. In lieu of imposing a single product on the market, lean asks the market what it values and answers to that demand. This is a crucial concept - and one that is often lost - when we ease lean into the health care system. When lean is discussed, the patient's notion of value is rarely the focus of the conversation. The founders at Toyota believed that customers craved variety. Traditional manufacturing machines and assembly lines efficiently produced a single product. The Toyota founders believed that by rearranging and reinventing the assembly line, they could increase the variety of the product they offered while simultaneously decreasing production costs. The key to this change lies in focusing on product flow through a system. Furthermore, one must ensure that the product flowing through the system is one born of the customer's perception of value. By focusing on what the end user values, the company is able to shed unnecessary manufacturing steps, decrease wasted time and energy, and produce something simultaneously less expensive to manufacture and of greater inherent consumer value.

\section{Terms with appeal}

Increased throughput, decreased costs, increased end-user perception of value. It does not take much to see why the promises of lean processing have worked their way into the hands of health care administrators. The leap to the medical world is not without its challenges and the need for amendment.

\section{Metaphorical challenges}

One of the toughest sells for lean is the analogy: an assembly line. The medical profession prides itself on valuing the individual and their problem as unique above all else. We categorize and label, but our work is never so mundane as to merit the comparison to a manufacturing line. This makes the assembly analogy inherent in the use of lean technology tricky at best. To that end, we must significantly bend the principles of lean, or pick and choose tools from the chest to give it value in our world of patient care.

That said, not all of the business of patient care involves direct interaction with the patient. While lean likely should not be adopted with the aim of treating the patient like a product pushed through the system, lean principles can be successfully used to decrease health care costs and improve care delivery.

\section{WASTE NOT, WANT NOT AND MEDICAL ERROR}

Remember that one of the founding principles of lean was waste reduction. In the manufacturing world, error is the principle source of waste. Take a machined automobile part as an example. Any deviation in shape or size may lead to mechanical failure. Manufacturers must throw away any parts that are incorrectly machined. This occurs after the investments of materials, energy and time have been made. Every manufactured throwaway increases the unit cost of the successful builds and the average manufacturing cost of the end product.

The lean approach, however, is not just as simple as focusing on waste output. The lean practitioner focuses on smoothing flow through the system first and foremost. The idea is that by improving flow, quality concerns are exposed and waste output falls.

The obvious analogy here is medical error. Unfortunately, medical errors have more dire consequences than tossed widgets and increased cost. The lean practitioner has a multitude of tools that have been developed over time to facilitate its system-wide implementation. Lean practitioners also work under a variety of philosophies to guide improvements. The notion of continuous improvement and the idea that error and waste arise from system failures is a good fit for attempting to lessen medical error.

${ }^{1}$ Division of Plastic Surgery, Department of Surgery, Queen's University, Kingston; ${ }^{2}$ Division of Plastic Surgery, Department of Surgery and Telfer School of Management, University of Ottawa, Ottawa, Ontario

Correspondence: Dr Douglas R McKay, Division of Plastic Surgery, Department of Surgery, Queen's University, Hotal Dieu Hospital, 166 Brock Street, Brock 3R, B3031, Kingston, Ontario K7L 5G2. Telephone 613-544-3400 ext 2494, fax 613-544-3709,

e-mail mckayd1@kgh.kari.net 


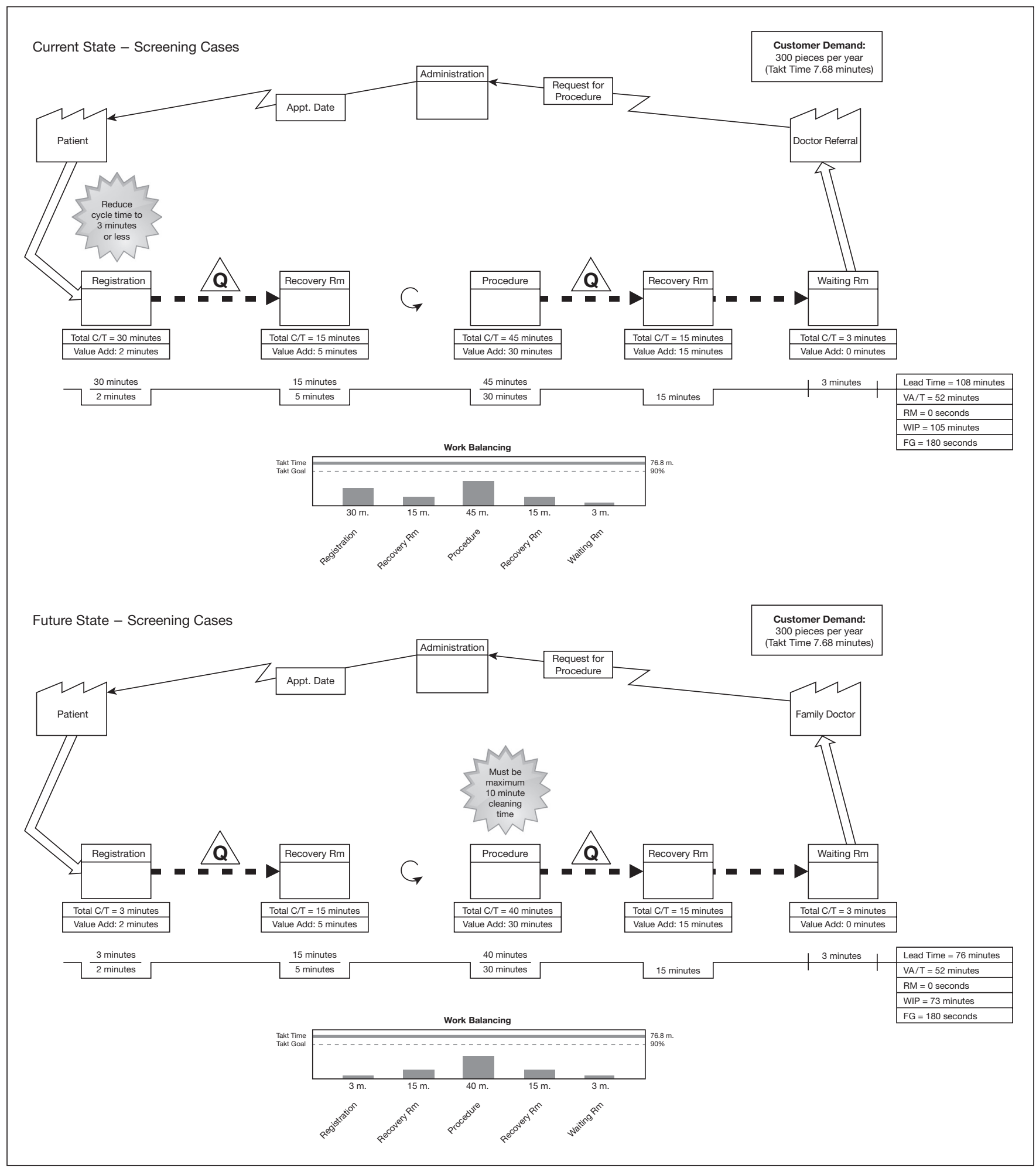

Figure 1) Not only does value stream mapping enable the administrator to study the problem in its entirety, it also easily identifies barriers to flow by tracking the time required to pass through the system. Major problems in process, such as a 30 min registration time, jump out as bottle necks when represented graphically. This found time can be used in a couple of ways: the obvious is increased throughput, but the less intuitive. Appt Appointment; C/T Cycle time; FG Finished goods; Rm Room; VA/T Value added time; WIP Work in process

A lean practitioner believes that error evolves out of system failures. Most of us can attest that medical error is no different. Occasionally, medical errors are pure mistakes, but most times, the explanation is not that simple. Most times, the error arises as a result of another shortcoming within the system. Hospitals are beginning to use the lean root-cause analysis tools to delve further in the nature of medical error. This analysis is further supported by adopting blame-free cultures of anonymous reporting to support the lean philosophy of continuous improvement.

Sadly, the initial driver of this style was not the altruistic notion of improved patient care but cost reduction. In the United States, Medicare and Medicaid stopped funding the treatment of preventable errors, forcing hospitals to assume the costs of quality deficiencies. 


\section{ADVANTAGES OF IMPROVED SYSTEM FLOW}

Although we are reticent to think of the patient as a cog in the system, there are certain advantages gleaned from improved throughput. One can argue back and forth whether throughput is a proxy for quality, but cannot deny that, at times, it carries advantage. If we put this debate aside for a moment and assume that a patient values a quick trip through the system, we can see that the lean approach is inherently advantageous in improving hospital flow. Certainly we can all imagine scenarios in which improved flow is advantageous to patients: waiting for a diagnosis or treatment in the emergency room; waiting for a trip to the operating room on the wait-and-see board; or getting in to see a specialist.

The original creators of lean focused their efforts on answering to customer value. Anything that produced value for the customer was called a value stream. A value stream is a series of processes or, in other words, a system that generates a product of desire. To study and improve a value stream, the technique of value stream mapping (VSM) was created to study process flow and is tremendously useful in improving health care flow. By creating a VSM, hospital administrators can study a variety of hospital issues in a wealth of detail to improve system flow (Figure 1).

As an example, take the colonoscopy suite at a hospital attempting to deal with the government mandated high-risk screening volumes while simultaneously treating patients with known disease. This is not an entirely random example and is, in fact, a problem the author and his MBA small group attempted to solve as part of their studies. To study the problem, a current state and future state VSM was created to demonstrate improvements in flow as a result of their proposed interventions.
Not only does the VSM allow the administrator to study the problem in its entirety it also easily identifies barriers to flow by tracking the time required to pass through the system. Major problems in process like a 30 minute registration time jump out as bottle necks when represented graphically. This found time can be used in a couple of ways. The obvious is increased throughput, but the less intuitive alternative is more time spent with patients either explaining the procedure beforehand, findings afterward, or time spent on the procedure itself.

\section{LEAN: A TOOL WITH POTENTIAL}

These are just a few of the ways lean can be used and implemented in order to improve patient care. The next time it is bandied around at a meeting, remember that there is much more to lean than cost control. The broader implications of its philosophy on health care delivery are far wider reaching. Every single facet of health care delivery has the potential to improve through lean process analysis.

ACKNOWLEDGEMENTS: Thanks to Trevor Braun MBA for creating the VSM used as an example and the Kingston Queens EMBA 2012 team.

\section{RESOURCES}

- Graban M. Lean Hospitals: Improving Quality, Patient Safety, and Employee Engagement, 2nd edn. New York: Productivity Press, 2011.

- Jimmerson C. Value Stream Mapping for Healthcare Made Easy. New York: Productivity Press, 2009.

- Lean Enterprise Institute <www.lean.org/whatslean/history.cfm> (Accessed April 12, 2015). 